This item was submitted to Loughborough's Research Repository by the author.

Items in Figshare are protected by copyright, with all rights reserved, unless otherwise indicated.

\title{
Shared-control for the kinematic model of a rear-wheel drive car
}

PLEASE CITE THE PUBLISHED VERSION

https://doi.org/10.1109/acc.2015.7170889

PUBLISHER

IEEE @ AACC

VERSION

AM (Accepted Manuscript)

LICENCE

CC BY-NC-ND 4.0

REPOSITORY RECORD

Jiang, Jingjing, and Alessandro Astolfi. 2019. "Shared-control for the Kinematic Model of a Rear-wheel Drive Car”. figshare. https://hdl.handle.net/2134/36761. 


\title{
Shared-Control for the Kinematic Model of a Rear-Wheel Drive Car
}

\author{
Jingjing Jiang ${ }^{1}$ and Alessandro Astolfi ${ }^{2}$
}

\begin{abstract}
This paper presents a shared-control algorithm for the kinematic model of a rear-wheel drive car, for which the set of feasible Cartesian positions is defined by a group of linear inequalities. The shared-control scheme is based on a hysteresis switch and its properties are established by a Lyapunov-like analysis. Simple numerical examples demonstrate the effectiveness of the shared-control law.
\end{abstract}

\section{INTRODUCTION}

This paper presents a solution to the shared-control problem for the kinematic model of a rear-wheel drive car. Here the name "shared-control" has the same meaning as in [1] and the shared-control used in anti-lock braking system. The human driver takes charge of the car in "normal" situations, while the feedback controller is "active" in "emergencies".

With the popularity of vehicles, many people rely on cars to go traveling, shopping and to work. In 2013, it is estimated that 74 per cent of all adults aged more than 17 in England hold a full car driving license [2]. Meanwhile, more than 1.7 thousands were killed and 181.9 thousands were injured in accidents on British Roads in 2013. Car occupants were the largest casualty type, accounting for 46 per cent of the death [3]. This shows a definite need for the development of automobiles that would help people drive safely. In this paper constraints on system states are used to describe safe operations of the car.

Researches started studying the autonomous driving problem by fuzzy logic controls [4], [5], [6] or linearized controls [7], [8]. Some of the works also combine fuzzy logic control with other algorithms, for example, sliding-mode controls [9], adaptive rules [10], PID controls [11], machine learning [12] or neural networks [13]. However, controllers designed for linearized models are suitable locally and fuzzy logic controls suffer from safety issues since no theoretical proof is given for system stability. This paper gives a shared-control algorithm to achieve the guarded motion globally together with theoretical proofs.

The obstacle avoidance problem has been studied in [14], where decentralized collision avoidance algorithms based on a switching strategy are presented. Systems controlled with this algorithm may undergo oscillations. The Virtual Force Field method is widely used in solving obstacle avoidance

\footnotetext{
${ }^{1}$ J. Jiang is with the Department of Electrical and Electronic Engineering, Imperial College London, UK, E-mail: jingjing.jiang10@imperial.ac.uk

${ }^{2} \mathrm{~A}$. Astolfi is with the Dept. of Electrical and Electronic Engineering, Imperial College London, London, SW7 2AZ, UK and the DICII, University of Roma "Tor Vergata", Via del Politecnico 1, 00133 Rome, Italy, E-mail: a.astolfieimperial.ac.uk
}

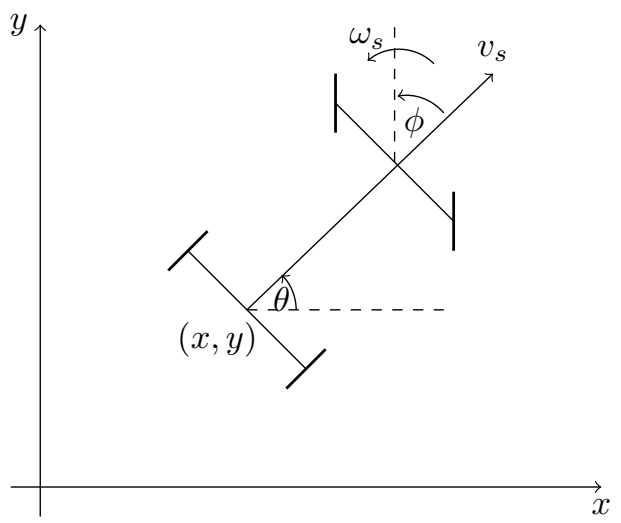

Fig. 1. Kinematic Model for a Rear-Wheel Drive Car

problems [15], [16]. However, the vehicle cannot reach the boundary of the obstacles even if the driver wants to stop next to the obstacle. Emergency stop is another effective way to avoid collisions [17], but the overall system stops rather than continuously moves. In [18], [19] networked complex intelligent transportation systems are studied by integration of cloud computing, agent programming and wireless data communication. GPS and expensive traffic management center are needed to build these systems. This paper proposes a novel continuous solution to the sharedcontrol problem which does not rely on communications between the vehicle and the management center or other vehicles. In addition, the vehicle with the shared-control can safely reach the boundaries of admissible configuration set.

The rest of the paper is organized as follows. In Section II the kinematic model of a rear-wheel drive car is given and the shared-control problem for the model is formulated. Some assumptions and a solution to the problem are given in Section III, in which formal properties of the closed-loop system with the shared-controller are presented. Section IV gives two numerical examples to illustrate the shared-control algorithm. Finally, some conclusions and ideas for future work are given in Section V.

\section{PROBLEM STATEMENT}

In this section we formulate the shared-control problem for the kinematic model of a rear-wheel drive car. Figure 1 shows the structure of the car, the dynamics of which are described 
by the equations

$$
\begin{aligned}
\dot{x} & =v_{s} \cos \theta, \\
\dot{y} & =v_{s} \sin \theta, \\
\dot{\theta} & =\frac{v_{s} \tan \phi}{l}, \\
\dot{\phi} & =\omega_{s},
\end{aligned}
$$

where $(x, y)$ denotes the Cartesian coordinates of the center of the rear-axle, $\theta$ is the heading angle of the car, $\phi$ represents the angle between the front-wheel and the heading direction of the car, $v_{s}$ and $\omega_{s}$ are the shared-control inputs of the system, describing the linear velocity of the car and the angular velocity of the front-wheels respectively. Note that $|\phi| \leq \frac{\pi}{2}-\epsilon$, with $0<\epsilon<\frac{\pi}{2}$, consistently to the physical construction of the car.

Let

$$
\begin{aligned}
& v_{s}(x, y, \theta, \phi, t)=\left[1-k\left(x, y, \theta, \phi, v_{h}\right)\right] v_{f}(x, y, \theta, \phi, t) \\
& +k\left(x, y, \theta, \phi, v_{h}\right) v_{h}(x, y, \theta, \phi, t), \\
& \omega_{s}(x, y, \theta, \phi, t)=\left[1-k\left(x, y, \theta, \phi, v_{h}\right)\right] \omega_{f}(x, y, \theta, \phi, t) \\
& +k\left(x, y, \theta, \phi, v_{h}\right) \omega_{h}(x, y, \theta, \phi, t),
\end{aligned}
$$

where $u_{h}=\left[v_{h}, \omega_{h}\right]^{T}$, denoted as $h$-control, describes the human action, $u_{f}=\left[v_{f}, \omega_{f}\right]^{T}$, denoted as $f$-control, represents the feedback-control action and $k$, denoted as sharing function, quantifies how the control action is shared.

In the rest of the paper, we assume that the h-control can be calculated from the pressure the driver applies to the accelerator and the torque applied to the steering wheel. We use the name s-closed-loop to denote the system described by equation (1) with the input given by equation (2) and the name $h$-closed-loop to denote the system described by the equations

$$
\begin{aligned}
& \dot{x}=v_{h} \cos \theta, \\
& \dot{y}=v_{h} \sin \theta, \\
& \dot{\theta}=\frac{v_{h} \tan \phi}{l}, \\
& \dot{\phi}=\omega_{h} .
\end{aligned}
$$

Note that the h-closed-loop system and the s-closed-loop system share the same state space $\mathcal{P} \times \mathcal{H} \times \mathcal{A}=\mathbb{R}^{2} \times \mathbb{S} \times \mathbb{A}^{1}$, where $\mathcal{P}$ is the set of Cartesian positions, $\mathcal{H}$ represents the set of heading angles and $\mathcal{A}$ denotes the set of angle differences between the front-wheel and the heading direction. Let $\mathcal{P}_{a} \in$ $\mathcal{P}$ be a given, connected and compact set describing the admissible Cartesian configuration set of the system (1) and $u_{h}$ be a given $\mathrm{h}$-control. Then the shared-control problem can be formulated as follows.

$$
{ }^{1} \mathbb{A} \text { is the set } \mathbb{A}=\left\{\phi \in \mathbb{R}^{n}:|\phi| \leq \frac{\pi}{2}-\epsilon\right\},
$$

with $0<\epsilon<\frac{\pi}{2}$.
Given the system (1), an admissible configuration set $\mathcal{P}_{a}$ and an h-control $u_{h}$, find (if possible)

- an f-control $u_{f}$;

- a sharing function $k$;

- a safe set $\mathcal{R}_{s}\left(v_{h}\right) \triangleq \mathcal{P}_{a} \times \mathcal{H}_{s} \times \mathcal{A}_{s} \subset \mathcal{P}_{a} \times \mathcal{H} \times \mathcal{A} \triangleq$ $\mathcal{R}\left(v_{h}\right)$;

such that the s-closed-loop system (1)-(2) has the following properties.

P1) The set $\mathcal{R}\left(v_{h}\right)$ is forward invariant.

P2) Let $\Omega_{s}$ and $\Omega_{h}$ be the $\Omega$-limit set of the s-closed-loop and h-closed-loop, respectively. Then

$$
\Omega_{s}= \begin{cases}\Omega_{h} & \text { if } \Omega_{h} \subset \mathcal{R}_{s}\left(v_{h}\right), \\ \Pi_{\mathcal{R}_{s}}\left(\Omega_{h}\right) & \text { if } \Omega_{h} \not \subset \mathcal{R}_{s}\left(v_{h}\right),\end{cases}
$$

where $\Pi_{\mathcal{R}_{s}}\left(\Omega_{h}\right)$ is the projection of $\Omega_{h}$ into the set $\mathcal{R}_{s}\left(v_{h}\right)$, which will be defined in Section III-A.

P3) $u_{s}=u_{h}$ if $(x, y, \theta, \phi) \in \mathcal{R}_{s}\left(v_{h}\right)$.

Note that for any fixed $v_{h}$ and any $(x, y) \in \mathcal{P}_{a}, \mathcal{H}_{s}$ and $\mathcal{A}_{s}$ are the sets of all possible heading angles and front-wheel angles such that the robot is unable to hit the boundary of $\mathcal{P}_{a}$ within a short time. In addition, this problem statement describes the case in which the operator has a feedback from the shared-controller (i.e. $u_{h}$ is a function of $k$ ), and the case in which the human does not have such feedback (i.e. $u_{h}$ is not a function of $k$ ).

Definition 1: The function $f(y(t), x(t), t)=$ $\operatorname{atg}(y(t), x(t), t)$ is a continuous function defined $\mathrm{as}^{2}$

$$
\operatorname{atg}(y(t), x(t), t)=\operatorname{atan}(y(t), x(t))+2 k(t) \pi,
$$

where atan is the four quadrant arctan function, $k(0)=0$ and

$$
k(t)= \begin{cases}k(t-\delta t)+1, & \text { if } a=-2 \pi \\ k(t-\delta t)-1, & \text { if } a=2 \pi \\ k(t-\delta t), & \text { else }\end{cases}
$$

with $a=\lim _{\delta t \rightarrow 0^{+}} \operatorname{atan}(y(t), x(t))-\operatorname{atan}(y(t-\delta t), x(t-\delta t))$ for all $t>0$.

\section{DESIGN OF THE SHARED-CONTROL FOR THE REAR-WHEEL DRIVE CAR}

In this section we give a solution to the shared-control problem given in Section II in the case in which the nonempty $\mathcal{P}_{a}$ is defined by a group of linear inequalities given by

$$
\mathcal{P}_{a}=\left\{p \in \mathbb{R}^{2} \mid S p+T \leq 0\right\},
$$

where $p=[x, y]^{T}, S=\left[s_{1}^{T}, s_{2}^{T}, \ldots, s_{m}^{T}\right]^{T} \in \mathbb{R}^{m \times 2}$ and $T=\left[t_{1}, t_{2}, \ldots, t_{m}\right]^{T} \in \mathbb{R}^{m}$. Similarly to [20] we make the following standing assumption.

\footnotetext{
${ }^{2}$ We use the 4 quadrant arctan function.
} 
Assumption 1: If $m>2$ then the matrices $S$ and $T$ are such that

$$
\operatorname{rank}\left(\left[\begin{array}{l}
s_{r_{1}} \\
\vdots \\
s_{r_{l}}
\end{array}\right]\right)<\operatorname{rank}\left(\left[\begin{array}{ll}
s_{r_{1}} & t_{r_{1}} \\
\vdots & \vdots \\
s_{r_{l}} & t_{r_{l}}
\end{array}\right]\right),
$$

for all $l \in[3, m]$ and $r_{1}, r_{2}, \ldots, r_{l} \in\{1,2, \ldots, m\}$.

\section{A. Design of the f-control}

This section gives a design of the feedback controller for the system (1) under the assumption that $m=2$. This is without loss of generality as detailed in [20]. Note that we need to design $N_{c}$ f-controls, each related to one group of active ${ }^{3}$ constraints. In general, $N_{c} \leq\left(\begin{array}{c}m \\ 2\end{array}\right)$. Consider the $i^{\text {th }}$ group of constraints

$$
S^{i} p+T^{i} \leq 0
$$

where $p=[x, y]^{T}, S^{i}=\left[s_{1}^{i^{T}}, s_{2}^{i^{T}}\right]^{T} \in \mathbb{R}^{2 \times 2}$ and $T=$ $\left[t_{1}^{i}, t_{2}^{i}\right]^{T} \in \mathbb{R}^{2}$ and define a new variable $q^{i}$ as

$$
q^{i}=S^{i} p+T^{i}
$$

System (1) with the associated constraints (4) controlled by a feedback-controller $u_{f}^{i}$ can be written, using $q^{i}$, as

$$
\begin{aligned}
& \dot{q}^{i}=S^{i}\left[\begin{array}{c}
\cos \theta^{i} \\
\sin \theta^{i}
\end{array}\right] v_{f}^{i}, \\
& \dot{\theta}^{i}=\frac{v_{f}^{i} \tan \phi^{i}}{l}, \\
& \dot{\phi}^{i}=\omega_{f}^{i}, \\
& q^{i} \leq 0 .
\end{aligned}
$$

To remove the constraint on $q^{i}$ we define new coordinates $z^{i}=\left[z_{1}^{i}, z_{2}^{i}\right]^{T}$, with

$$
z_{j}^{i}=\log \frac{q_{j}^{i}}{q_{r_{j}}^{i}}
$$

for all $j \in\{1,2\}$, where $q_{r_{j}}^{i}$ is the reference trajectory relative to $q_{j}^{i}$ and is defined as

$$
q_{r_{j}}^{i}= \begin{cases}q_{d}^{i}, & \text { if } q_{d_{j}}^{i} \leq\left(1-\frac{\sqrt{2}}{2}\right) r-\epsilon \\ -\epsilon, & \text { if } q_{d_{j}}^{i} \geq(\sqrt{2}-1) r-\epsilon \\ h_{j}^{i}, & \text { otherwise }\end{cases}
$$

for $j \in\{1,2\}$, where $r$ is a positive constant, $h_{j}^{i}=-(r+$ $\epsilon)+\sqrt{r^{2}-\left[(\sqrt{2}-1) r-\epsilon-q_{d_{j}}^{i}\right]^{2}}, q_{d}^{i}=\left[q_{d_{1}}^{i}, q_{d_{2}}^{i}\right]^{T}=$ $S^{i} p_{d}+T^{i}$ and $p_{d}$ describes the desired trajectory in the space $\mathcal{P}$. In the trajectory tracking case, the feedback controller and the driver share the same information of the reference signal, i.e. $q_{d}^{i}$ is known to the feedback controller. On the other hand, in a free-driving case, i.e. no given trajectory to track, $q_{d}^{i}(t)$ is calculated from $u_{h}(t)$. In other words, the

${ }^{3}$ For each velocity $v$ and heading angle $\theta$, the $j^{\text {th }}$ constraint is active if

$$
\exists k>0: k s_{j}[v \cos \theta, v \sin \theta]^{T}+t_{j}=0 .
$$

human behaviour is regarded as the reference behaviour by the feedback controller. Note that $q_{r_{j}}^{i}$ is a smooth function taking only negative values. Using $q_{r}^{i} p_{r}^{i}, \alpha_{r}^{i}, v_{r}^{i}, \theta_{r}^{i}, \phi_{r}^{i}$ and $\omega_{r}^{i}$ are depicted as

$$
\begin{aligned}
p_{r}^{i} & =S^{i^{-1}}\left(q_{r}^{i}-T^{i}\right), \\
\alpha_{r}^{i} & =S^{i-1} \dot{q}_{r}^{i}, \\
v_{r}^{i} & =\sqrt{\alpha_{r_{1}}^{i}{ }^{2}+\alpha_{r_{2}}^{i}{ }^{2}}, \\
\theta_{r}^{i} & =\operatorname{atan} 2\left(\alpha_{r_{2}}^{i}, \alpha_{r_{1}}^{i}\right), \\
\phi_{r}^{i} & =\operatorname{atan}\left(\frac{\dot{\theta}_{r}^{i} l}{v_{r}^{i}}\right), \\
\omega_{r}^{i} & =\dot{\phi}_{r}^{i} .
\end{aligned}
$$

Let $\left(p_{d}, \theta_{d}, \phi_{d}\right) \in \Omega_{h}$ and define the projection of $\left(p_{d}, \theta_{d}, \phi_{d}\right)$ into $\mathcal{R}_{s}\left(v_{h}\right)$ relative to the $i^{\text {th }}$ group of active constraints, i.e. $\Pi_{\mathcal{R}_{s}}^{i}\left(p_{d}, \theta_{d}, \phi_{d}\right)$, as

$$
\Pi_{\mathcal{R}_{s}}^{i}\left(p_{d}, \theta_{d}, \phi_{d}\right)=\left(p_{r}^{i}, \theta_{r}^{i}, \phi_{r}^{i}\right),
$$

where $\left(p_{r}^{i}, \theta_{r}^{i}, \phi_{r}^{i}\right)$ is given by (8). Then the projection of $\Omega_{h}$ into $\mathcal{R}_{s}\left(v_{h}\right)$ relative to the $i^{\text {th }}$ group of constraints is defined by

$$
\Pi_{\mathcal{R}_{s}}^{i}\left(\Omega_{h}\right)=\left\{s \in \mathcal{R}_{s} \mid s=\Pi_{\mathcal{R}_{s}}^{i}\left(p_{d}, \theta_{d}, \phi_{d}\right)\right\},
$$

for all $\left(p_{d}, \theta_{d}, \phi_{d}\right) \in \Omega_{h}$. With the use of the new variable $z^{i}$, system (5) can be rewritten as

$$
\begin{aligned}
& \dot{z}_{1}^{i}=\frac{v_{f}^{i} \cos \theta^{i}}{e^{z_{1}^{i}} q_{r_{1}}^{i}}-\frac{v_{r}^{i} \cos \theta_{r}^{i}}{q_{r_{1}}^{i}}, \\
& \dot{z}_{2}^{i}=\frac{v_{f}^{i} \cos \theta^{i}}{e^{z_{2}^{i}} q_{r_{2}}^{i}}-\frac{v_{r}^{i} \cos \theta_{r}^{i}}{q_{r_{2}}^{i}}, \\
& \dot{\theta}^{i}=\frac{v_{f}^{i} \tan \phi^{i}}{l} \\
& \dot{\phi}^{i}=\omega_{f}^{i} .
\end{aligned}
$$

Let

$\begin{aligned} & \theta^{i *}=\operatorname{atg}\left(e^{z_{2}^{i}}\left(v_{r}^{i} \sin \theta_{r}^{i}-\gamma_{2} z_{2}^{i}\right), e^{z_{1}^{i}}\left(v_{r}^{i} \cos \theta_{r}^{i}-\gamma_{1} z_{1}^{i}\right)\right), \\ & \phi^{i *}=\operatorname{atan}\left(\begin{array}{c}l \frac{z_{1}^{i}}{e^{z_{1}^{i}} q_{r_{1}}^{i}} \sin \frac{\theta+\theta^{i *}}{2} \operatorname{sinc} \frac{\theta-\theta^{i *}}{2}+\frac{\theta^{i *} l}{v_{f}^{i}} \\ -l \frac{z_{2}^{i}}{e^{z_{2}^{i}} q_{r_{2}}^{i}} \cos \frac{\theta+\theta^{i *}}{2} \operatorname{sinc} \frac{\theta-\theta^{i *}}{2}\end{array}\right), 1\end{aligned}$

where $\gamma_{1}>0, \gamma_{2}>0$.

Consider the $i^{\text {th }}$ Lyapunov function $L^{i}\left(z_{1}^{i}, z_{2}^{i}, \theta^{i}, \phi^{i}\right)$ given by

$$
L^{i}\left(z_{1}^{i}, z_{2}^{i}, \theta^{i}, \phi^{i}\right)=\frac{1}{2}\left\{\begin{array}{c}
z_{1}^{i^{2}}+z_{2}^{i^{2}}+\left(\theta^{i}-\theta^{i *}\right)^{2} \\
+\left(\tan \phi^{i}-\tan \phi^{i *}\right)^{2}
\end{array}\right\},
$$

and choose $u_{f}^{i}=\left[v_{f}^{i}, \omega_{f}^{i}\right]^{T}$ such that $L^{i}<0$ for all $\left(z_{1}^{i}, z_{2}^{i}, \theta^{i}, \phi^{i}\right) \neq\left(0,0, \theta^{i *}, \phi^{i *}\right)$. One such choice is given 
by

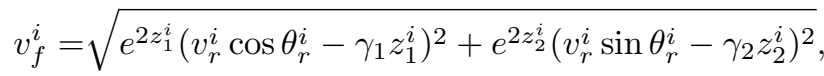

$$
\begin{aligned}
& \omega_{f}^{i}=\cos ^{2} \phi^{i}\left[\begin{array}{c}
-\frac{v\left(\theta^{i}-\theta^{i *}\right)}{l}+\frac{\dot{\phi}^{i *}}{\cos ^{2} \phi^{i *}} \\
-\gamma 3\left(\tan \phi-\tan \phi^{i *}\right)
\end{array}\right],
\end{aligned}
$$

which yields

$$
\dot{L}^{i}=\gamma 1 \frac{z_{1}^{i^{2}}}{q_{r_{1}}^{i}}+\gamma 2 \frac{z_{2}^{i^{2}}}{q_{r_{2}}^{i}}-\gamma 3\left(\tan \phi-\tan \phi^{*}\right)^{2} \leq 0 .
$$

This can be pushed back into the $(p, \theta, \phi)$ coordinates by

$$
\begin{aligned}
& v_{f}^{i}=\sqrt{\begin{array}{c}
\left(\frac{q_{1}^{i}}{q_{r_{1}}^{i}}\right)^{2}\left(v_{r}^{i} \cos \theta_{r}^{i}-\gamma_{1} \log \frac{q_{1}^{i}}{q_{r_{1}}^{i}}\right)^{2} \\
+\left(\frac{q_{2}^{i}}{q_{r_{2}}^{i}}\right)^{2}\left(v_{r}^{i} \sin \theta_{r}^{i}-\gamma_{2} \log \frac{q_{2}^{i}}{q_{r_{2}}^{i}}\right)^{2}
\end{array}} \\
& \omega_{f}^{i}=\cos ^{2} \phi^{i}\left[\begin{array}{c}
-\frac{v\left(\theta^{i}-\theta^{i *}\right)}{l}+\frac{\dot{\phi}^{i *}}{\cos ^{2} \phi^{i *}} \\
-\gamma 3\left(\tan \phi-\tan \phi^{i *}\right)
\end{array}\right],
\end{aligned}
$$

where

$$
\begin{gathered}
\phi^{i *}=\operatorname{atan}\left(\begin{array}{c}
l \frac{\log \frac{q_{1}^{i}}{q_{r_{1}}^{i}}}{q_{1}^{i}} \sin \frac{\theta+\theta^{i *}}{2} \operatorname{sinc} \frac{\theta-\theta^{i *}}{2}+\frac{\theta^{i *} l}{v_{f}^{i}}, 1 \\
-l \frac{q_{2}^{i}}{q_{2}^{i}} \cos \frac{\theta+\theta^{i *}}{q_{r_{2}}} \operatorname{sinc} \frac{\theta-\theta^{i *}}{2}
\end{array}\right), \\
\theta^{i *}=\operatorname{atg}\left(\begin{array}{c}
\frac{q_{2}^{i}}{q_{r_{2}}^{i}}\left(v_{r}^{i} \cos \theta_{r}^{i}-\gamma_{2} \log \frac{q_{2}^{i}}{q_{r_{2}}^{i}}\right), \\
\frac{q_{1}^{i}}{q_{r_{1}}^{i}}\left(v_{r}^{i} \cos \theta_{r}^{i}-\gamma_{1} \log \frac{q_{1}^{i}}{q_{r_{1}}^{i}}\right)
\end{array}\right),
\end{gathered}
$$

and $q_{j}^{i}=s_{j}^{i} p+t_{j}^{i}, q_{r_{j}}^{i}=s_{j}^{i} p_{r}+t_{j}^{i}$ for all $j \in\{1,2\}$.

Lemma 1: Consider the system (1) controlled by the feedback controller (11) where $q_{r}^{i}$ is defined by (7), $v_{r}^{i}$ and $\theta_{r}^{i}$ are defined by (8). Suppose $\mathcal{P}_{a}$ is described by (3) and $(x(0), y(0)) \in \mathcal{P}_{a}$. Then the closed-loop system has the following properties.

- $(x(t), y(t)) \in \mathcal{P}_{a}$ for all $t \geq 0$;

- $\lim _{t \rightarrow \infty}\left(x(t)-p_{r_{1}}(t)\right)=\lim _{t \rightarrow \infty}\left(y(t)-p_{r_{2}}(t)\right)=0$.

Remark 1: One of the feedback control input $\omega_{f}^{i}$ given by (11) may be large (due to sharp changes of $\phi^{i *}$ ). A bound for $\omega_{f}^{i}$ can be determined using Algorithm 1 given on the top of this page. Note that $\left|\omega_{f}^{i}\right|>B$ only occurs when the Cartesian position of the car is close to the boundary of the admissible configuration set and the feedback controller becomes active.

\section{B. Shared Control Theorem}

Similarly to [21] we need to determine the safe subset $\mathcal{R}_{s}$ before giving the shared-control law. Relative to each given h-control and $i^{\text {th }}$ group of constraints, the set $\mathcal{R}$ can be

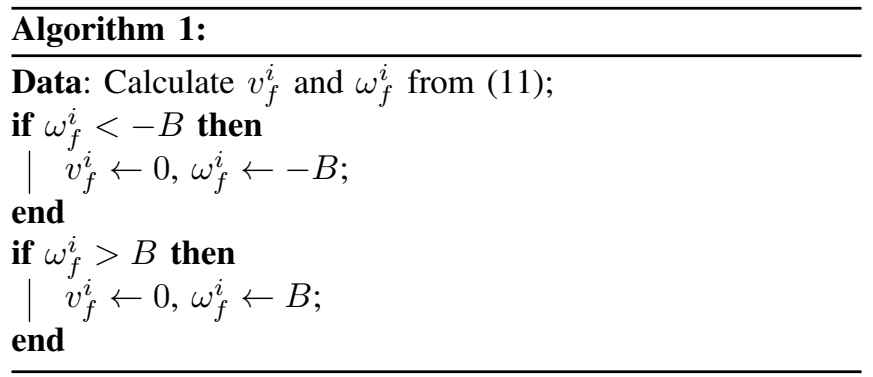

divided into three subsets $\tilde{\mathcal{R}}_{s}, \tilde{\mathcal{R}}_{h}$ and $\tilde{\mathcal{R}}_{d}$ by equations (13), given on the top of next page, where $\mathcal{Q}_{a}^{i}=S^{i} \mathcal{P}_{a}+T^{i}$, and $b_{2}>b_{1}>0$. Note that this definition is given in the $\left(q^{i}, \theta^{i}, \phi^{i}\right)$ coordinates and can be translated to the $(x, y, \theta, \phi)$ coordinates by the relations

$$
\begin{aligned}
& \mathcal{R}_{s}^{i}\left(v_{h}\right)=\operatorname{diag}\left(\mathrm{S}^{\mathrm{i}-1}, \mathrm{I}\right)\left(\tilde{\mathcal{R}}_{\mathrm{s}}^{\mathrm{i}}-\operatorname{col}\left(\mathrm{T}^{\mathrm{i}}, 0\right)\right), \\
& \mathcal{R}_{h}^{i}\left(v_{h}\right)=\operatorname{diag}\left(\mathrm{S}^{\mathrm{i}-1}, \mathrm{I}\right)\left(\tilde{\mathcal{R}}_{\mathrm{h}}^{\mathrm{i}}-\operatorname{col}\left(\mathrm{T}^{\mathrm{i}}, 0\right)\right), \\
& \mathcal{R}_{d}^{i}\left(v_{h}\right)=\operatorname{diag}\left(\mathrm{S}^{\mathrm{i}-1}, \mathrm{I}\right)\left(\tilde{\mathcal{R}}_{\mathrm{d}}^{\mathrm{i}}-\operatorname{col}\left(\mathrm{T}^{\mathrm{i}}, 0\right)\right),
\end{aligned}
$$

where $\operatorname{col}\left(\mathrm{T}^{\mathrm{i}}, 0\right)$ is a column vector obtained by stacking the zero vector 0 under the vector $T^{i}$. Note that the subsets $\mathcal{R}_{s}^{i}\left(v_{h}\right), \mathcal{R}_{h}^{i}\left(v_{h}\right)$ and $\mathcal{R}_{d}^{i}\left(v_{h}\right)$ have the same properties as those given in [21].

We can now define the sharing function relative to the $i^{\text {th }}$ group of constraints $k^{i}$ as

$$
k^{i}\left(p, \theta, v_{h}\right)= \begin{cases}1, & (p, \theta, \phi) \in \mathcal{R}_{s}^{i}\left(v_{h}\right) \backslash \mathcal{R}_{d}^{i}\left(v_{h}\right), \\ l^{i}, & (p, \theta, \phi) \in \mathcal{R}_{h}^{i}\left(v_{h}\right), \\ 0, & (p, \theta, \phi) \in \mathcal{R}_{d}^{i}\left(v_{h}\right),\end{cases}
$$

where

$$
l^{i}= \begin{cases}1, & \text { if }(p, \theta, \phi) \text { enters } \mathcal{R}_{h}^{i}\left(v_{h}\right) \text { from } \mathcal{R}_{s}^{i}\left(v_{h}\right) \\ 0, & \text { if }(p, \theta, \phi) \text { enters } \mathcal{R}_{h}^{i}\left(v_{h}\right) \text { from } \mathcal{R}_{d}^{i}\left(v_{h}\right)\end{cases}
$$

Finally, the shared-control input is given as

$$
\begin{aligned}
u_{s}\left(p, \theta, \phi, v_{h}\right)= & \sum_{i=1}^{N_{c}}\left[\left(1-k^{i}\left(p, \theta, v_{h}\right)\right) u_{f}^{i}\left(p, \theta, \phi, p_{r}, \theta_{r}, \alpha_{r}\right)\right] \\
& +\min _{i=1}^{N_{c}} k^{i}\left(p, \theta, v_{h}\right) u_{h} .
\end{aligned}
$$

Proposition 1: Consider the kinematic model of a rear-wheel drive car (1) with the shared-control input (11)-(14)-(15). Let $\mathcal{P}_{a}$ be a given closed, connected, admissible configuration set defined by (3) and $u_{h}$ be a given h-control. Assume $(x(0), y(0)) \in \mathcal{P}_{a}$. Then there exist positive $\gamma_{1}, \gamma_{2}, \gamma_{3}$ and $b_{2}>b_{1}>0$ such that the s-closed-loop system has the following properties.

(1) $(x(t), y(t)) \in \mathcal{P}_{a}$ for all $t \geq 0$.

(2) $\Omega_{s}=\Pi_{\mathcal{R}_{s}}\left(\Omega_{h}\right)$.

(3) $u_{s}(t)=u_{h}(t)$ for all $t \geq 0$ and $(p(t), \theta(t), \phi(t)) \in$ $\mathcal{R}_{s}\left(v_{h}(t)\right) \backslash \mathcal{R}_{d}\left(v_{h}(t)\right)$. 


$$
\begin{gathered}
\tilde{\mathcal{R}}_{s}^{i}\left(v_{h}\right)=\left\{\left(q^{i}, \theta^{i}, \phi^{i}\right) \in \mathcal{Q}_{a}^{i} \times \mathbb{S} \times \mathbb{A}:\left(s_{j}^{i}\left[\cos \theta^{i}, \sin \theta^{i}\right]^{T} v_{h}\right) \leq \frac{1}{q_{j}^{i}+b_{2}}-\frac{1}{b_{2}} \text { if } q_{j}^{i} \geq-b_{2} \text { for all } j \in\{1,2\}\right. \\
\tilde{\mathcal{R}}_{h}^{i}\left(v_{h}\right)=\left\{\begin{aligned}
\left(q^{i}, \theta^{i}, \phi^{i}\right) \in \mathcal{Q}_{a}^{i} \times \mathbb{S} \times \mathbb{A}: & \exists j \in\{1,2\} \text { such that }\left(s_{j}^{i}\left[\cos \theta^{i}, \sin \theta^{i}\right]^{T} v_{h}\right)>\frac{1}{q_{j}^{i}+b_{2}}-\frac{1}{b_{2}} \text { and } q_{j}^{i} \geq-b_{2} \\
& \text { and }\left(s_{k}^{i}\left[\cos \theta^{i}, \sin \theta^{i}\right]^{T} v_{h}\right)<\frac{1}{q_{k}^{i}+b_{1}}-\frac{1}{b_{1}} \text { if } q_{k}^{i} \geq-b_{1} \text { for all } k \in\{1,2\}
\end{aligned}\right\} \\
\tilde{\mathcal{R}}_{d}^{i}\left(v_{h}\right)=\left\{\begin{aligned}
\left(q^{i}, \theta^{i}, \phi^{i}\right) \in \mathcal{Q}_{a}^{i} \times \mathbb{S} \times \mathbb{A}: & \exists j \in\{1,2\} \text { such that }\left(s_{j}^{i}\left[\cos \theta^{i}, \sin \theta^{i}\right]^{T} v_{h}\right) \geq \frac{1}{q_{j}^{i}+b_{1}}-\frac{1}{b_{1}},-b_{1} \leq q_{j}^{i}<0 \\
& \text { or } \exists j \in\{1,2\} \text { such that }\left(s_{j}^{i}\left[\cos \theta^{i}, \sin \theta^{i}\right]^{T} v_{h}\right)>\frac{1}{q_{j}^{i}+b_{1}}-\frac{1}{b_{1}}, q_{j}^{i}=0 \\
& \text { or } \forall j \in\{1,2\} \text { such that } q_{j}^{i}=\left(s_{j}^{i}\left[\cos \theta^{i}, \sin \theta^{i}\right]^{T} v_{h}\right)=0
\end{aligned}\right\}
\end{gathered}
$$

Proposition 2: Consider the system (1) with the sharedcontrol input (11)-(14)-(15). Suppose $\phi(0) \in\left(-\frac{\pi}{2}, \frac{\pi}{2}\right)$. Then $|\phi(t)|<\frac{\pi}{2}$ for all $t \geq 0$.

\section{NUMERICAL EXAMPLES}

This section discusses two numerical examples: one for trajectory tracking and the other for free driving. Note that the car without the shared-controller goes outside of the admissible configuration set in both cases.

\section{A. Trajectory Tracking}

Consider the kinematic model of the rear-wheel drive car given by equation (1) and the admissible configuration set defined by

$$
\mathcal{P}_{a}=\{(x, y) \mid x \geq 0, y \leq 5\}
$$

Assume the reference trajectory is a circle with radius 2 and centered at $(1,2.5)$, i.e.

$$
p_{d}(t)=[2 \cos (0.05 t)+1,2 \sin (0.05 t)+2.5]^{T} .
$$

Simulation results are shown in Figure 2 and 3. Note that at the beginning of the simulation the trajectory of the s-closedloop coincides with that of the h-closed-loop, matching the fact that $u_{s}=u_{h}$ when $t<29$. $u_{s}$ differs from $u_{h}$ at $t=29$, when the car is close to the bound $x=0$. After $110 s$, the time history of $u_{s}$ overlaps that of $u_{h}$ again since the car is sufficiently away from the boundaries of $\mathcal{P}_{a}$. Note that the shared-control input is bounded, $v_{s} \geq 0$ and $\omega_{s} \in[-1,1]$.

\section{B. Free Driving}

Consider the system (1) again with the $\mathcal{P}_{a}$ defined by (16). Let the human drive in a haphazard way yielding the $(x, y)$ trajectory displayed by the red, dashed-and-dotted, curve in Figure 4 . The trajectory of the s-closed-loop system is given by the green, dashed, curve. The path resulting from the $\mathrm{h}-$ closed-loop enters the non-admissible region (gray, shaded), while that with the shared-control remains in $\mathcal{P}_{a}$, thus indicating the effectiveness of the shared-control algorithm.

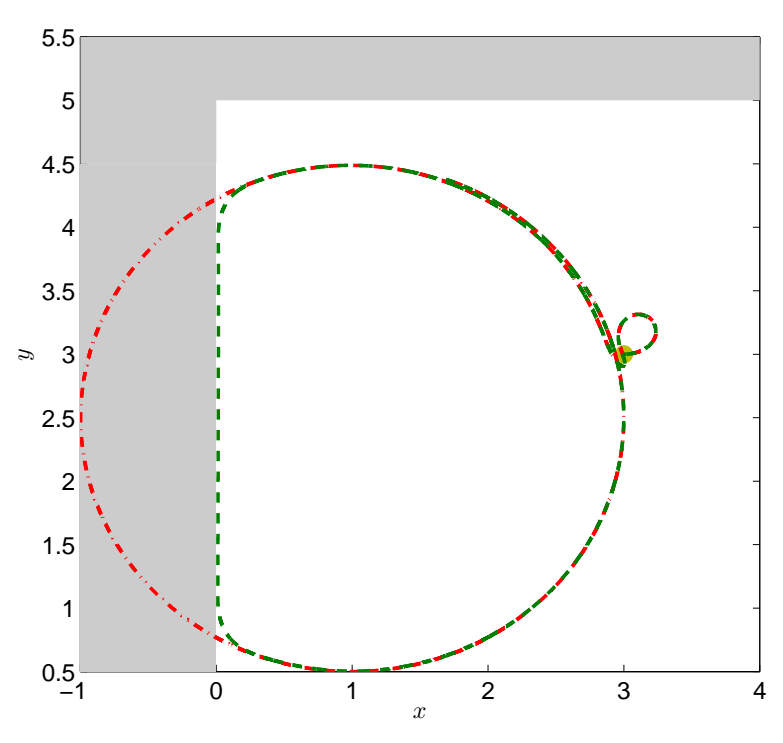

Fig. 2. $(x, y)$ trajectories of the system (1) for the set $\mathcal{P}_{a}$ given in (16): h-closed-loop (red, dashed-and-dotted) and s-closed-loop (green, dashed). Round mark: the initial position of the car.

In addition, the $(x, y)$ trajectory of s-closed-loop overlaps with that of the h-closed-loop again when the car is far away from the boundary of $\mathcal{P}_{a}$ and the state of the system enters the safe subset $\mathcal{R}_{s}$.

\section{CONCLUSIONS}

We have presented a solution to the shared-control problem for the kinematic model of a rear-wheel drive car. The algorithm is based on a hysteresis switch to integrate the human input and the feedback control action. The sharing weight is determined by the sets $\mathcal{R}_{s}\left(v_{h}\right), \mathcal{R}_{h}\left(v_{h}\right)$ and $\mathcal{R}_{d}\left(v_{h}\right)$. Two simple numerical examples given in Section IV demonstrate the effectiveness of the shared-control law. Future work will be devoted to 3D UAVs and nonlinear mechanical systems. 


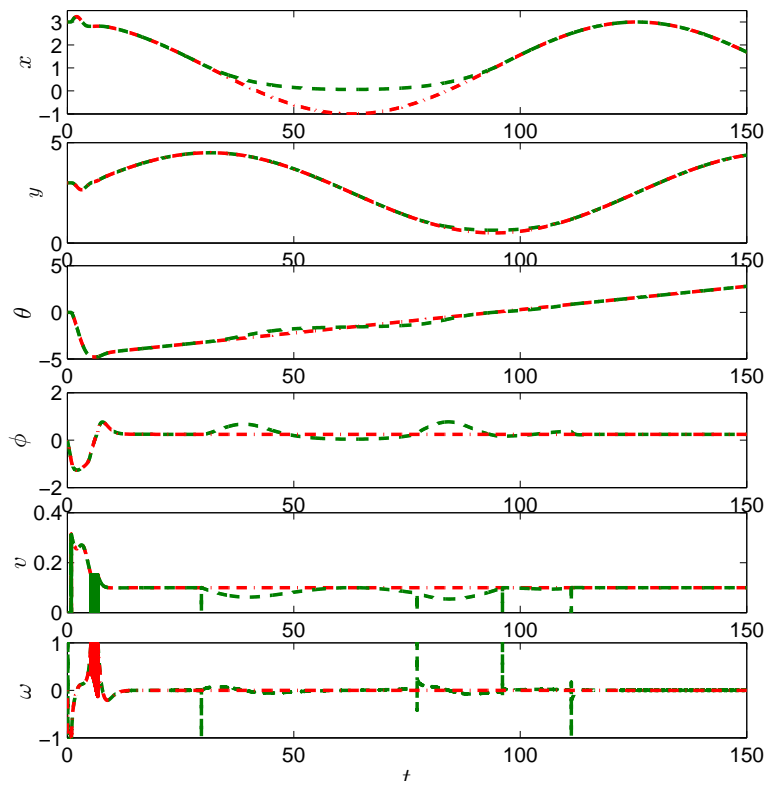

Fig. 3. Time histories of the variable $x, y, \theta, \phi, v$ and $\omega$ for the h-closed-loop system (red, dashed-and-dotted) and s-closed-loop system (green, dashed).

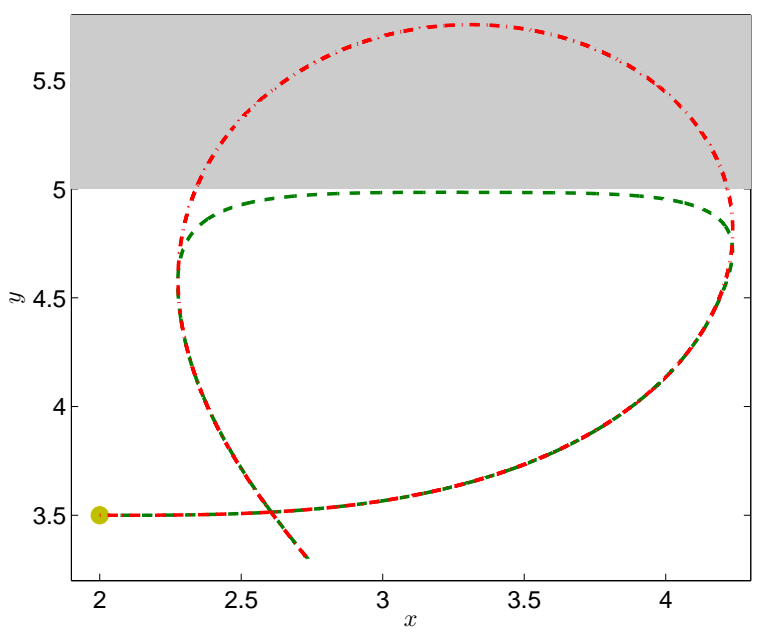

Fig. 4. $(x, y)$ trajectories of the system (1) for the set $\mathcal{P}_{a}$ given in (16): h-closed-loop (red, dashed-and-dotted) and s-closed-loop (green, dashed). Round mark: the initial position of the car.

\section{REFERENCES}

[1] T. Rofer and A. Lankenau, "Ensuring safe obstacle avoidance in a shared-control system," in Proc. of IEEE International Conference on Emerging Technologies and Factory Automation, vol. 1, Barcelona, 1999, pp. 1405-1414.

[2] D. for Transport, "National travel survey: England 2013," 2014.

[3] W. H. Organization, 2014.

[4] Y. Li, S. Chang, and Y. Chen, "Implementation of human-like driving skills by autonomous fuzzy behavior control on an fpga-based car-like mobile robot," IEEE Trans. on Industrial Electronics, vol. 50, no. 5, pp. 867-880, 2003.
[5] I. Baturone, F. Moreno-Velo, S. Sanchez-Solano, and A. Ollero, "Automatic design of fuzzy controllers for car-like autonomous robots," IEEE Trans. on Fuzzy Systems, vol. 12, no. 4, pp. 447-465, 2004.

[6] T. Li and S. Chang, "Autonomous fuzzy parking control of a car-like mobile robot," IEEE Trans. on Systems, Man and Cybernetics, Part A: Systems and Humans, vol. 33, no. 4, pp. 451-465, 2003.

[7] A. Luca, G. Oriolo, and C. Samson, "Feedback control of a nonholonomic car-like robot," Robot Motion Planning and Control, vol. 229, pp. 171-253, 1998

[8] H. Sira-Ramiirez and R. Castro-Linares, "Trajectory tracking for nonholonomic cars: A linear approach to controlled leader-follower formation," in Proc. of IEEE Conference on Decision and Control, 2010, pp. 546-551.

[9] C. Hwang, L. Chang, and Y. Yu, "Network-based fuzzy decentralized sliding-mode control for car-like mobile robots," IEEE Trans. on Industrial Electronics, vol. 54, no. 1, pp. 574-585, 2007.

[10] S. Kim and J. Kim, "Adaptive fuzzy-network-based c-measure mapmatching algorithm for car navigation system," IEEE Trans. on Industrial Electronics, vol. 48, no. 2, pp. 432-441, 2001.

[11] H. Abatari and A. Tafti, "Using a fuzzy pid controller for the path following of a car-like mobile robot," in Proc. of RSI/ISM International Conference on Robotics and Mechatronics, 2013, pp. 189-193.

[12] J. Lin and R. Lian, "Intelligent control of active suspension systems," IEEE Trans. on Industrial Electronics, vol. 58, no. 2, pp. 618-628, 2011

[13] T. Li, C. Chen, and K. Lim, "Combination of fuzzy logic control and back propagation neural networks for the autonomous driving control of car-like mobile robot systems," in Proc. of SICE Annual Conference, 2010, pp. 2071-2076.

[14] G. Hoffmann and C. Tomlin, "Decentralized cooperative collision avoidance for acceleration constrained vehicles," in Proc. of IEEE Conference on Decision and Control, 2008, pp. 4357-4363.

[15] S. Boonyarak and P. Prempraneerach, "Real-time obstacle avoidance for car robot using potential field and local incremental planning," in Proc. of SICE Annual Conference, 2010, pp. 2071-2076.

[16] F. Ding, J. Peng, X. Bian, and H. Wang, "AUV Local Path Planning Based on Virtual Potential Field," in Proc. of IEEE Conference on Mechatronics and Automation, 2005, pp. 1711-1716.

[17] E. Coelingh and S. Solyom, "All aboard the robotic road train," IEEE Spectrum, vol. 49, no. 11, pp. 34-39, 2012.

[18] F. Wang, "Parallel control and management or intelligent transportation systems: Concepts, architectures, and applications," IEEE Trans. on Intelligent Transportation Systems, vol. 11, no. 3, pp. 630-638, 2010

[19] M. Padmadas, K. Nallaperumal, V. Mualidharan, and P. Ravikumar, "A deployable architecture of intelligent transportation system a developing country perspective," in Proc. of IEEE Conference on Computational Intelligence and Computing Research, 2010, pp. 1-6.

[20] J. Jiang and A. Astolfi, "Shared-control for fully actuated linear mechanical systems," in Proc. of IEEE Conference on Decision and Control, Florence, Italy, 2013, pp. 4699-4704.

[21] - "Shared-control for the kinematic model of a mobile robot," in Proc. of IEEE Conference on Decision and Control, Los Angeles, USA, 2014, pp. 62-67. 